\title{
A Study of the Impact of APTS on Service Quality Perceptions of Elderly and Disabled Riders
}

\author{
Julian M. Benjamin \\ North Carolina A\&T State University
}

\begin{abstract}
New transportation technology that directly impacts consumers should be evaluated by the people who are affected. Automated dispatching has become standard practice for paratransit services. This article summarizes a study analyzing consumer response to the Mobility Manager at a demonstration site in Winston-Salem, North Carolina.

The Mobility Manager was applied to the TransAID demand-responsive mini-bus service for people who are elderly or who have disabilities. Survey data from two questionnaires, before and after the implementation of the Mobility Manager for the same subjects, were used to examine travel behavior and perceived service quality. These travelers reported service improvements such as easier telephone access and shorter travel times. The respondents' travel patterns after implementation of the Mobility Manager remained stable. This article also provides econometric estimates of the change in the number of trips as a function of the change in travel attributes affected by implementation of the Mobility Manager. Changes in the number of trips by survey respondents were treated as a Poisson random variable. Results from a Poisson regression show that the primary beneficiaries of the Mobility Manager were riders with disabilities. Perceived service attributes that significantly affected changes in trips were length of trip, number of stops picking up additional passengers, and physical comfort.
\end{abstract}




\section{Study Background}

The Advanced Public Transportation Systems (APTS) program of the Federal Transit Administration in the 1990s involved projects that demonstrated the application of advanced technologies to transit systems (Casey et al. 1991). The APTS program evaluated these new technologies in practical demonstration projects. The system selected as the site for this study was the mini-bus dial-a-ride for special populations in Winston-Salem, North Carolina. As one of the APTS demonstration sites, new transit technologies including automated computer dispatch, automatic vehicle location, and smart cards were tested and evaluated. Taken together, these technologies made up the Mobility Manager, a geographic information system combined with a management information system. The Mobility Manager assisted the transit agency in scheduling, routing, billing, and administration tasks.

The technology was tested in a mini-bus system called TransAID, which was part of the public transit system of Winston-Salem (WSTA). Winston-Salem is a small city (2000 U.S. census population of 182,874) and is part of the Piedmont Triad, which consists of three cities with similar size about 30 miles apart in north central North Carolina. TransAID is a public paratransit system with curb-to-curb dial-aride service.

TransAID services are provided in eight 15-passenger mini-buses (vans) that are equipped for non-disabled passengers and 11 vans equipped for wheelchairs. Weekday hours of operation in Winston-Salem and in Forsyth County are 5:30 A.M. to 6:30 P.M. Limited service is provided for dialysis patients on Saturdays.

\section{Study Design}

The evaluation of this project used procedures summarized in "Evaluation Guidelines for the Advanced Public Transportation Systems Operational Tests" (Casey and Collura 1993). These techniques were based on methodologies developed for the earlier UMTA demonstration program. Subsequently, there have been many advances in travel analysis procedures that have particular relevance to project evaluations. These findings were summarized by Benjamin (1994) and included travel activity diaries, stated preference techniques applied to complex decisions, the application of computer technology to data gathering, and the application of new econometrics procedures. This study investigated the application of many of these procedures to the evaluation of APTS by applying them in a case study. 
Results of the stated and revealed preference elements of the before study were reported in Ben-Akiva et al. (1996). These study methods are combined into a before and after study design in this article.

\section{Study Objectives}

The objectives of this study were twofold. First, the study provided before and after comparisons of perceived service quality improvements from using the WSTA Mobility Manager. These improvements include various aspects perceived by users including telephone answering, travel time, and impacts on consumer travel behavior. Second, we considered the decision by TransAID passengers to take additional trips to determine the extent to which the implementation of Mobility Manager induced more ridership via the enhancement of service attributes.

\section{Before and After Surveys}

The before survey was completed in the summer of 1994 just before the phase 1 demonstration project started. The survey was designed to collect both baseline evaluation data and consumer demand information from current riders. The survey consisted questions in four areas: current travel behaviors, current transit system performance, stated anticipated travel behaviors after the APTS was implemented, and subject demographics.

The after study took place two years later following implementation of the Mobility Manager and replicated the before questions except for the stated preference travel questions.

Current travel behavior was recorded during the week prior to contact with the respondent. Detailed questions were asked about travel by trip purpose for each day of the week. Trip making for an entire one week period was investigated because of the low trip rate by the elderly and persons with disabilities; 78 percent of these respondents reported in the before study that they traveled by TransAID only once during the study week.

Questions on current transit service quality consisted of measures of the method used to contact TransAID to reserve a trip to and from home, responsiveness of the telephone service, and vehicle travel time. Each question asked about the last time that TransAID was used so that measures of service quality could be averaged across subjects. These questions were tailored to the anticipated improvements in the quality of service by the Mobility Manager, which would make it more efficient to schedule trips and make travel time shorter as a result of more efficient routing. 
Questions on future responses included scenarios that represented different hypothetical services. In anticipation of service improvements by the Mobility Manager, questions addressed how far in advance vans must be reserved, the ability to confirm calls immediately, travel time, and time required to call for return trips after the Mobility Manager was placed in to operation.

\section{Respondent Descriptions \\ Response Rate and Attrition}

The before study questionnaire was completed by 266 respondents. Of this total, 176 respondents were reported to still be TransAID riders by the Winston-Salem Transit Authority (WSTA) at the time of the after study. For the after study, the 176 subjects were initially contacted by mail. Current phone numbers were obtained for 162 respondents, and 100 surveys were completed. The 62 subjects who did not respond consisted of:

- 12 for whom there was no answer after five callbacks,

- 8 who had passed away,

- 14 who reported that they no longer use TransAID,

- 13 whose phone numbers were disconnected,

- 9 who did not live at the most recent phone numbers,

- 2 whose phone numbers had been changed to nonpublished numbers,

- 2 who had no recollection of their last trip on TransAID, and

- 2 who refused to participate.

\section{General Socioeconomic Statistics}

The initial data analysis was presented by Benjamin (2000). During the before study, TransAID served people with disabilities ( $2 / 3$ of the survey respondents), children under 12 years of age (30 respondents), and adults 65 years of age or older (162 respondents). The largest percentage of attrition occurred for younger riders (e.g., 50 riders before, 2 riders after), which may be due in part to HeadStart children entering public schools. The next largest attrition rate was for those over 65 years of age and was partly due to mortality and to changes in dwelling places for this age group.

Most people reported having a high school education in both studies, with the highest attrition rate for those reporting only elementary education. This, again, 
may have been due to the HeadStart youngsters. Among all the users, only 5 out of 272 respondents were employed in the before study and only 2 in the after study. Among those reporting to have a disability in the before study, 95 were sight impaired, 101 were hearing impaired, 81 noted that the nature of their disability was "difficulty in reaching and grasping," 139 had difficulty walking, and 83 used a wheelchair. In the after study, the largest group had difficulty walking (65 of 101).

\section{Service Usage and Quality Before Mobility Manager}

Van Service Usage Patterns for Initial Respondents. The larger number of the responses before the Mobility Manager provided information for a more detailed analysis of service utilization and quality. In the before study, 45 percent of the respondents rode TransAID the week prior to the first survey but only 30 percent rode it during the second survey. The trips reported were unequally distributed among days of the week. The largest number traveled on Monday (47\%) with other trips distributed among the remaining days of the week. Only 2 percent of the sample reported riding on Saturday, and there was no service provided on Sunday. Similar results were reported in the second survey. (These travel patterns are illustrated for the last trip in Table 3.)

In a summary of trips made according to disability type and trip purpose, for the first trip taken, 76 percent of these users traveled for medical reasons. The majority of people rode for medical reasons in each disability group in the before and after studies, and the disability group with the largest percentage of medical trips was for those who had difficulty walking. Virtually all of the trips were round-trips, and most people traveled only once during the week. The average number of one-way trips reported during the survey week was 3.6. About one third of the respondents made a second round-trip, and about one fourth made more trips. Thirty-seven subjects made five round-trips, and only one subject reported making a sixth trip (for medical purposes) before and, at most, five round-trips after.

\section{Service Quality Before Mobility Manager}

All questions about quality of service referred to the last time the service was used. This section summarizes responses before implementation of the Mobility Manager. Almost 80 percent of the respondents said that the last time they called for service they did not get a busy signal, only 14 percent felt rushed, 5 percent complained of lack of courtesy, and 6 percent reported a lack of accuracy. Respon- 
dents reported talking an average of 2.8 minutes and holding for a mean of about 1 minute.

The vehicle usually arrived on time (65\%), although the vehicles were frequently either early (14\%) or late (20\%). The mean time of early pick-up was 3.9 minutes; the mean late pick-up time was 8 minutes. However, 29 percent of the riders indicated that the vehicle had failed to pick them up at some time in the past. For the last return trip, the van arrived at the destination within 5 minutes of schedule 200 times but there was a reported late arrival of one hour or more by 17 people.

Riders reported an average in-vehicle travel time of 22.2 minutes, with a standard deviation of 22.1 minutes. While traveling, respondents observed an average of 4 people in their vans. When they arrived early, it was only for an average of 1.5 minutes; when the vehicle arrived late, the average reported time was 9.1 minutes, with a standard deviation of 25.6 minutes.

During the week before the first survey, 26 percent of the respondents had ridden the fixed-route transit an average of 1.4 times, indicating that there could be some shift to the fixed-route mode. Sometime in the past, 10 percent of the respondents reported that they had been in an accident on the vehicle and that assistance was required. It took an average of 34 minutes for assistance to arrive, with two hours being the longest wait.

\section{Van Service Usage Patterns}

Practically all the trips (more than $95 \%$ ) taken by children under 18 in the survey group were for educational (other) purposes. This proportion is unique to this age group, and adults between 18 and 65 years old also had a significant amount (about 20\%) of educational trips. That the youngest group has such a high proportion of educational trips fits in with the fact that TransAID was designed in part to serve families with children attending HeadStart programs. Van service reservation patterns for this age group indicate that for close to 60 percent of the trips taken, the van service was not called, implying that the service was scheduled in advance and worked like a school bus that picks up at home. For the remaining 40 percent, the service was requested a minimum of 24 hours in advance. As in the other age groups, the proportion of users reserving the van the same day as the scheduled trip was negligible ( $0 \%$ for children under 12 , under $5 \%$ for all the other respondents).

For respondents above 65 years old, at least 60 percent of the trips were made for medical purposes. More than 50 percent of the trips were reserved 24 hours in 
advance. Other trip purposes for this group were for shopping and nutrition, each representing close to 10 percent of the trips, indicating that TransAID was helping with some of their daily activities.

\section{Comparison of Before and After Surveys}

A comparison of results for those who took both surveys was calculated for key questions in the study. The HeadStart children in the first phase all aged out of using these services before the second phase and were therefore not included in any analysis based on the after data. Table 1 lists some responses to key questions asked before the Mobility Manager and responses by the same people in the after survey.

\section{Table 1. Perceived Effects of APTS}

\begin{tabular}{|l|l|c|}
\hline Reported Before & Reported After by Same Respondents & After Percent \\
\hline Picked up early/late & Picked up on time & $21 \%$ \\
\hline Picked up early/late & Same & $13 \%$ \\
\hline & Lower travel time & $57 \%$ \\
\hline Dropped off late & Same & $37 \%$ \\
\hline $\begin{array}{l}\text { Called more than 24 hours in } \\
\text { advance }\end{array}$ & Same & $80 \%$ \\
\hline & Lower phone time & $57 \%$ \\
\hline Not polite on phone & Same & $90 \%$ \\
\hline Busy signals & More busy signals & $80 \%$ \\
\hline
\end{tabular}

The extent the implementation of the Mobility Manager favorably impacted service quality perceptions by riders was not absolutely definitive. The following sample proportions, with t-statistics in parentheses, suggest that the implementation of the Mobility Manager may have impacted favorably on service quality perceptions. Of those respondents reporting that TransAID last picked them up early or late in the first survey, 21 percent $(t=5.1)$ reported that TransAID last picked them up on time in the second survey, 5 percent $(t=2.3)$ reported that TransAID last picked them up early in the second survey, and 8 percent $(t=2.9)$ reported that TransAID last picked them up late in the second survey. Given the 
travel times reported in the first survey, 57 percent $(t=11.5)$ of the respondents in the second survey reported spending less time riding TransAID from their home. For respondents who reported in the first survey that were dropped off at their destination late, only 37 percent $(t=7.6)$ reported that TransAID dropped them off late in the second survey. Of those respondents in the first survey reporting that they did not request their last trip on TransAID at least 24 hours in advance, 80 percent $(t=20)$ reported scheduling their last trip at least 24 hours in advance in the second survey. For reported times spent talking to TransAID officials on the telephone in the first survey, 57 percent $(t=11.5)$ reported spending less time talking in the second survey. Of those respondents in the first survey who reported that TransAID officials were not polite on the telephone, 90 percent $(t=$ 30) reported they were polite in the second survey.

Although fewer people reported traveling on TransAID in the second survey, of those who rode TransAID in the first survey, 85 percent reported traveling slightly more frequently by TransAID after the Mobility Manager. However, a chi-square test revealed that there was no significant difference for the total group in trip rates for different trip purposes and days of the week.

To the extent that perceptions of quality were related to the attributes measured in the sample proportions cited above, one implication was that the Mobility Manager had a favorable impact. Such an implication was, of course, only suggested by the reported sample proportions. Further caution was warranted by the fact that there was some evidence suggesting that perceptions of service quality were lower for respondents in the second survey in some areas. For example, of those respondents in the first survey reporting the number of busy signals when attempting to contact TransAID officials on the telephone, 80 percent $(t=20)$ reported an increase in the number of busy signals in the second survey.

\section{Effects of Mobility Manager on Ridership}

\section{Trips as a Function of Travel Attributes}

Presumably, consumer response to the implementation of Mobility Manager should have resulted in an increased number of trips. If, for example, consumers, as utility maximizers, realized higher utility as a result of enhanced travel attributes, the extent to which the implementation of Mobility Manager enhanced travel attributes on TransAID should induce more ridership. Five questions in the followup survey were designed to measure travel attributes such as ease of scheduling, 
physical comfort, and trip length. Each question provided answers on a five-point integer scale, with 1 indicating strong agreement and 5 indicating strong disagreement. ${ }^{1}$

Both surveys recorded the number of trips on TransAID by respondents. To determine the effect of the Mobility Manager on ridership, we posit the relationship between the change in number of trips and the service attributes plausibly affected by Mobility Manager as follows:

$\Delta$ Trips $=\mathrm{T}_{\mathrm{i}}=f($ Travel Attributes $)$

$$
=f(\text { Easy, Long, Stops, Riders, Comfort })
$$

where:

Easy

is the extent to which it was easy to schedule a trip on TransAID

Long

represents the extent to which it takes long to complete a trip on TransAID

Stops indicates the extent to which TransAID makes many stops for other passengers

Riders signifies the extent to which there were too many passengers on TransAID

Comfort is the extent to which a passenger was comfortable while riding TransAID

All variables were based on the perception of the respondent in the second survey, after implementation of the Mobility Manager.

The rate of incidence of the number of trips was modeled as the arrival rate in a random Poisson process. This modeling approach is discussed in the Supplemental Material section.

\section{Results}

Table 2 presents the mean and standard deviation for the dependent and independent variables. Column 1 in Table 3 reports the Poisson regression estimates 
of equation (1). ${ }^{2}$ As a goodness-of-fit measure, Table 3 also reported the value of Pseudo- $\mathrm{R}^{2}{ }^{3}$ All of the parameter estimates conformed to a priori expectations and were significant except for the variables Riders and Comfort. Column 1 also reports the estimated coefficient $\alpha$, which was generated from testing equation (1) for mean variance equality, a restriction required for a Poisson random variable. ${ }^{4}$ The insignificance of $\alpha$ at any reasonable level of significance implied that $T_{i}$ could have been modeled as a Poisson random variable. The sign on Easy suggested that the more difficult scheduling a trip was on TransAID, the higher the probability of additional ridership by a respondent. This, of course, seems rather implausible, but it may capture the effects of riders willing to tolerate difficulty scheduling as a result of it being positively correlated with other favorable attributes associated with TransAID after the implementation of the Mobility Manager. Long was negative and significant, suggesting that the probability of ridership increased with decreases in trip duration. The positive and significant sign on Stops indicated that the probability of ridership increased as the number of passenger pick-ups decreased. The insignificance of Rider and Comfort suggested that the probability of ridership changes was not affected at all by perceptions of the number of passengers and their comfort.

Table 2. Summary Statistics

\begin{tabular}{lccccc}
\hline Variable & Mean & $\begin{array}{c}\text { Standard } \\
\text { Deviation }\end{array}$ & Minimum & Maximum & N \\
\hline $\begin{array}{l}\text { Number of } \\
\text { Additional Trips }\end{array}$ & & & & & \\
$\left(\mathrm{T}_{\mathrm{i}}\right)$ & 1.46 & 4.74 & 0 & 33 & 99 \\
Easy & 1.78 & .767 & 1.0 & 5.0 & 99 \\
Long & 2.28 & .858 & 1.0 & 5.0 & 99 \\
Stops & 3.24 & .959 & 1.0 & 5.0 & 99 \\
Riders & 4.12 & .824 & 1.0 & 5.0 & 99 \\
Comfort & 1.0 & 1.11 & 1.0 & 5.0 & 99 \\
\hline
\end{tabular}

Note: Two observations were lost due to survey respondents not responding to the questions quiring a categorical response 
Table 3. Poisson and Negative Binomial Regression Estimates

\begin{tabular}{|l|l|l|l|l|}
\hline Variable & $(1)$ & $(2)$ & $(3)$ & $(4)$ \\
\hline Constant & .498 & .254 & .209 & .192 \\
& $(.626)$ & $(.412)$ & $(.752)$ & $(.153)$ \\
\hline Easy & -.276 & -.193 & -.627 & -.432 \\
& $(.148)^{*}$ & $(.112)^{*}$ & $(.178)^{* * *}$ & $(.234)^{*}$ \\
\hline Long & -.306 & -.257 & -.384 &. .223 \\
& $(.113)^{* * *}$ & $(.129)^{* *}$ & $(.122)^{* * *}$ & $(.163)$ \\
\hline Stops & 2.22 & 1.35 & 2.82 & 1.75 \\
& $(.303)^{* * *}$ & $(.783)^{*}$ & $(.325)^{* * *}$ & $(.521)^{* * *}$ \\
\hline Riders & .002 & .002 & .067 & .032 \\
& $(.105)$ & $(.157)$ & $(.127)$ & $(.148)$ \\
\hline Comfort & .037 & .032 & .300 & .248 \\
& $(.108)$ & $(.234)$ & $(.121)^{* * *}$ & $(.127)^{*}$ \\
\hline Pseudo-R & .231 & .284 & .293 & .302 \\
\hline Sample Size & 99 & 99 & 83 & 83 \\
\hline$\alpha$ & 4.93 & $\ldots$ & 3.32 & $\ldots$ \\
& $(3.62)$ & $\ldots .$. & $(3.02)$ & $\ldots$ \\
\hline V & $\ldots .$. & 1.02 & $\ldots$ & 1.37 \\
& & $(.95)$ & & $(1.03)$ \\
\hline NR & $\ldots$ & .063 & $\ldots$ & .054 \\
\hline$\chi^{2}$ & $\ldots$ & .095 & $\ldots$ & .073 \\
\hline
\end{tabular}

Note: Standard errors were in parentheses.

* Significant at the .10 level.

** Significant at the .05 level.

*** Significant at the .01 level.

The adequacy of the Poisson specification in equation (1) for modeling additional trips on TransAID suggested that the implementation of the Mobility Manager engendered additional trips by enhancing the utility of riders as a result of shorter trips (Long), and a fewer number of stops per trip picking up other passengers (Stops).

Given that approximately 84 percent of the sample consisted of TransAID riders who self-reported some type of disability, the insignificance of the variables Riders and Comfort could have reflected a failure to control for the handicap status of TransAID riders. To explore this possibility, a discriminate analysis was conducted to determine which of the TransAID riders' handicap status can discriminate between those respondents who reported taking more trips on TransAID in the 
second survey and those who did not. The discriminant analysis revealed that the handicap status of TransAID riders did indeed provide explanatory power for discriminating between two groups, with handicapped riders more likely to report taking additional trips on TransAID in the second survey.

Column 3 in Table 3 shows the results of a Poisson regression estimated for the sample of riders who self-reported having a disability. As was the case for the Poisson specification in column 1 , the sign and significance of $\alpha$ and $\chi^{2}$ for testing Poisson specification in column 3 against the negative binomial in column 4 , suggests the adequacy of the Poisson specification over the sample of riders self-reporting a disability. The results in column 3 differed from the results in column 1 in that the variable Comfort was positive and significant. The variable Riders was still positive but insignificant. Apparently for riders with disabilities, the level of comfort was proportional to the number of passengers on TransAID. Thus, to the extent that the Mobility Manager was able to reduce the number of passengers per trip on TransAID, it induced additional trips by TransAID riders with disability. For public transit riders with disabilities that restricted mobility and/or required visits for medical care, it seems reasonable that ease of scheduling and physical comfort would have been important factors determining the desirability of public transit. Thus, the results reported in Table 3 suggest that the Mobility Manager, to the extent that it improved the attributes associated with travel on TransAID, and increased ridership, did so for disabled passengers with disabilities by enhancing the passenger utility attributes of trip length, number of stops picking up other passengers, and rider comfort.

\section{Conclusions}

The new technology used by TransAID directly affected consumers, and it was that effect that is reported in this study. The van service was used primarily by people above 65 years old to take medical trips ( $1 / 3$ of all trips reported in the survey). The use of the TransAID vehicles in part reflects the preferences and constraints of the riders and reflects trip purpose, weekday, and time-of-day restrictions by funding agencies.

Several key findings resulted from the survey. First, there was substantial attrition. The total attrition here was 63 percent. Of these subjects, there were only two who refused to participate (less than 1\%). Attrition may be due to changes in travel behavior over time, substitution of other modes, or the transient nature 
of this population. The remaining people included a large number who moved or changed phone numbers. This suggests that future research on new transit technologies, such as the Mobility Manager, should over sample the relevant population.

Second, the results of the second survey suggested that implementation of the Mobility Manager in Winston-Salem improved customer satisfaction with stable ridership on TransAID. The data suggest that the Mobility Manager impacted favorably on perceived service quality evidenced by shorter travel times, improved customer service through fewer telephone difficulties such as being put on hold, and decreased late drop-offs. However, overall ridership decreased because of attrition, but riders reported stable individual use of the service between the two surveys. Restrictions from funding agencies may have combined with consumer preferences and constraints for limited changes in travel patterns.

The Poisson regression results suggest that the Mobility Manager enhanced the attractiveness of travel by TransAID. For respondents with disabilities who highly rated comfort and ease of service, the Mobility Manager increased the use of TransAID.

Reactions of other travelers who began riding TransAID during the study period were assumed to be similar. Results were viewed in light of the sampling restrictions and the small population size. The results reported here must be interpreted as findings from retrospective questions. Although questions were asked about the last trip taken and the last week, the ability to recall details may have been limited for some members of these special groups. Finally, this case study suggests that the potential usefulness of a technology such as the Mobility Manager, from the consumers' perspective, is its ability to improve the perceived quality of service. Given the linkage between perception of service quality and ridership, future research on new transit technologies should explore which technologies will best increase ridership. 


\section{Appendix}

As the number of trips was reported as a count variable in both surveys, we specify the change in the number of trips $\left(T_{i}\right)$ as a Poisson random variable:

$$
\operatorname{Prob}\left(T_{i}=n\right)=f(T i)\left[\exp \left(-\lambda_{i}\right) \lambda_{i}^{n}\right] / n !
$$

where:

$T_{i}$ is the additional number of trips on TransAID reported by respondent $I$

$n$ equals $0,1,2, \ldots . \mathrm{N}$

$e \quad$ is 2.71828

$\lambda_{i} \quad$ represents the expected value of $T_{i}=$ Variance of $T_{i}$

A regression model was formulated by specifying the Poisson parameter $\lambda_{i}$ as a deterministic function of the presumed exogenous variables Easy, Long, Stops, Riders, and Comfort, with an unknown parameter vector $\beta$. We estimate the following specification:

$$
\lambda i=\exp \left[\beta_{0}+\beta_{1} \text { Easy }+\beta_{2} \text { Long }+\beta_{3} \text { Stops }+\beta_{4} \text { Riders }+\beta_{5} \text { Comfort }\right]
$$

A Poisson specification such as equation (1) was a member of the class of Generalized Linear Models (GLM), the parameters will be estimated with a nonlinear weighted least squares maximum likelihood procedure. ${ }^{5}$ The log likelihood for the number of additional trips $\mathrm{T}_{\mathrm{i}}$ is:

$$
L(\beta)=\Sigma\left[T_{i} !-\exp \left(X_{i} \beta\right)+T_{i} X_{i} \beta\right]
$$

where:

$X_{i} \quad$ is a vector of exogenous variables

The gradient and Hessian, respectively, are:

$$
\begin{aligned}
& \partial L(\beta) / \partial \beta=\Sigma\left[X_{i}^{\prime}\left(T_{i}-\exp \left(X_{i} \beta\right)\right)\right]=0 \\
& \partial^{2} L(\beta) / \partial \beta \partial \beta^{\prime}=\Sigma\left[-X_{i}^{\prime} X_{i} \exp \left(X_{i} \beta\right)\right]<0
\end{aligned}
$$


The first order condition was nonlinear in $\beta$ and can be estimated with a numerical maximum likelihood procedure, or by an iterative nonlinear least squares procedure. Because the Hessian was negative definite, convergence to a unique solution was assured.

To further assess the explanatory power and adequacy of a Poisson regression specification for additional trips by passengers on TransAID, column 2 of Table 4 reported and tested the results of a Poisson specification versus a negative binomial specification of equation (1). ${ }^{6}$ The negative binomial specification was tested as an alternative to the Poisson specification with a Lagrange Multiplier Test, where the Poisson specification was viewed as the restricted model. ${ }^{7}$ The insignificance of the $\chi^{2}$ for the Lagrange Multiplier Test statistic $N R^{2}$ in column 2 indicates that the restricted Poisson regression specification cannot be rejected against the negative binomial alternative.

\section{Endnotes}

${ }^{1}$ The questions related to the Regression Model specifications were as follows:

a. It was easy to schedule a trip on TransAID.

b. It does not take long to complete a trip on TransAID.

c. TransAID made many stops picking up other passengers.

d. There were too many passengers on TransAID vehicles.

e. I am physically comfortable when riding TransAID.

${ }^{2}$ All results were obtained with the use of LIMDEP (Version 7.0) Econometric Software.

${ }^{3}$ Specifically, the goodness-of-fit measure was the Pseudo- $R^{2}$ of McFadden (1974) defined as $R^{2}=1-\left(\log L_{\Omega}\right) /\left(\log L_{\omega}\right)$, where $L_{\Omega}$ was the maximum of the likelihood function when maximized with respect to all of the parameters, and $L_{\omega}$ was the maximum of the likelihood function when maximized with respect to a constant term only.

${ }^{4}$ If dependent variable Ti has a Poisson distribution, the mean of the dependent variable $T_{i}$ was $\lambda_{i}=\lambda_{i}\left(X_{i}, \beta\right)$. Cameron and Trivedi (1990) show that a test for mean-variance equality was based on the hypothesis test: $H_{0}: \operatorname{var}\left(T_{i}\right)=\lambda_{i}$ versus the alternative: $H_{A}: \operatorname{var}\left(T_{i}\right)=\lambda_{i}+\alpha g\left(\lambda_{i}\right)$, where $g\left(\lambda_{i}\right)$ was a function specified to equal 
$1, \lambda_{i}$, or $\lambda_{i}{ }^{2}$. A test for mean-variance equality was a t-test for the significance of $\alpha$ in the auxiliary regression: $\Sigma w_{i} g\left(\lambda_{i}\right)\left\{\left(T_{i}-\lambda_{i}\right)^{2}-T_{i}-\alpha g\left(\lambda_{i}\right)\right\}=0$, where $\Sigma w_{i} g\left(\lambda_{i}\right)$ was a weight based on a consistent estimate of $\beta$-the fitted value for example. If the $t$-test on the coefficient $\alpha$ was insignificant, the null hypothesis of mean-variance equality cannot be rejected, suggesting the adequacy of a Poisson specification for the dependent variable-additional number of trips on TransAID.

${ }^{5}$ See Nelder and Wedderburn (1972) and Price (1995).

${ }^{6} \mathrm{~A}$ negative binomial random variable can be viewed as a realization of random variable from a specific compound Poisson distribution (Cameron and Trivedi 1986), where the mean varies linearly with the variance-or there was overdispersion. The overdispersion parameter in a negative binomial regression can be obtained from an auxiliary regression of the form $\left[\left(T_{i}-\lambda_{i}^{2}\right] / \lambda_{i}-1=v \lambda_{i}+k_{i}\right.$, where $v$ was the overdispersion parameter, and $k_{i}$ was a heteroskedastic stochastic error term. A negative binomial regression specification results when the estimate of $v_{i}$, was added as a variable to the Poisson regression specification.

${ }^{7}$ The Lagrange Multiplier Test procedure was based on an auxiliary regression where the residuals of the Poisson regression were regressed against all the variables initially included in the Poisson specification plus the estimated overdispersion parameter $v$. This was an approach suggested by Engle (1982), and we implement it here by viewing the Poisson specification as a restricted model, and the negative binomial specification as an unrestricted model. The test-statistic, distributed as a chi-square $\left(\chi^{2}\right)$ with degrees of freedom equal to the difference in the number of parameters between the negative binomial and Poisson regression specifications, was determined by the product of the unadjusted $R^{2}$ from the auxiliary regression times the number of observations $(N)$. If $N x R^{2}$ exceeds the critical value of the chi-square statistic, the null hypothesis that the overdispersion parameter $v$ has a zero coefficient that would have to be rejected.

\section{Acknowledgments}

This project was funded in part by the Urban Transit Institute of North Carolina A\&T State University, which was funded in part by U.S. Department of Transportation. Valuable research assistance was provided by William Nelson, a student in the transportation program at North Carolina A\&T State University. We also thank the manager of the Winston-Salem Transit Authority, Nedra Woodyethe, and the manager of TransAID, Suzanne Telechea, for their assistance with this 
project and Professor John Stone of NC State University for his assistance with the before study questionnaire. We also thank Dr. Gregory Price for his assistance with the data analysis.

\section{References}

Ben-Akiva, M., J. Benjamin, G. Lauprete, and A. Polydoropolou. 1996. Impact of Advanced Public Transportation Systems on Travel by Dial-a-Ride. Transportation Research Record 1557: 72-77.

Benjamin, J. 1994. Current trends in data gathering. Final Report. Prepared for the USDOT/FHWA.

Benjamin, J. 1995. Initial data analysis of the phase 1 before survey. Unpublished memorandum.

Cameron, A. C., and P. K. Trivedi. 1986. Econometric models based on count data: Comparisons and applications of some estimators. Journal of Applied Econometrics 1: 29-53.

Cameron, A. C., and P. K Trivedi. 1990. Regression based tests for overdispersion in the Poisson model. Journal of Econometrics 46: 347-364.

Casey, R., and J. Collura. 1993. Evaluation guidelines for the Advanced Public Transportation Systems operational tests. USDOT/FTA Report Number FTAMA-26-0007-94-2.

Casey, R., L. Labell, S. Prensky, and C. Schweiger. 1991. Advanced public transportation systems: The state of the art. USDOT/FTA Report No. DT-VNTSC-UMTA91-2.

Engle, Robert F. 1982. A general approach to Lagrangian Multiplier diagnostics. Annals of Econometrics, 20: 83-104.

LIMDEP 7.0: User's manual and reference guide. 1995. Econometric Software Inc., Bellport, NY.

McFadden, Daniel. 1974. The measurement of urban travel demand. Journal of Public Economics 3: 303 -328.

Nelder, J. A., and R. W. Wedderburn. 1972. Generalized linear models. Journal of the Royal Statistical Society 135: 370-384. 
Price, G. N. 1995. The determinants of entry for black-owned commercial banks. Quarterly Review of Economics and Finance 35: 289-302.

\section{About the Author}

JULIAN BeNJAmin (benjamin@ncat.edu) is a professor in the Department of Economics and Transportation/Logistics at North Carolina A\&T State University. He received his Ph.D. from the Department of Civil Engineering at the State University of New York at Buffalo. His research has focused on special transportation and travel demand analysis. He has many publications in these areas with several that look at the application of demand analysis to planning services in a special transportation agency. He is a former member of the GATE (an early special transportation agency) Board of Directors. 